\title{
Optimized analysis method for indirect dark matter searches with Imaging Air Cherenkov Telescopes
}

\author{
Jelena Aleksić ${ }^{* \dagger}$ \\ Institut de Física d'Altes Energies (IFAE), E-08193 Barcelona, Spain \\ E-mail: jelena@ifae.es \\ Javier Rico \\ IFAE \\ E-mail: jricoeifae.es \\ Manel Martínez \\ IFAE \\ E-mail: martinez@ifae.es
}

\begin{abstract}
We describe a dedicated analysis approach for indirect Dark Matter searches with Imaging Air Cherenkov Telescopes. By using the full likelihood analysis, we take complete advantage of the distinct features expected in the gamma ray spectrum of Dark Matter origin, achieving better sensitivity with respect to the standard analysis chains. We compare its sensitivity with that of the current standards for several Dark Matter annihilation models, obtaining gains of up to factors of order of 10. We compute the improved limits that can be reached using this new approach, taking as an example the recent results from VERITAS on observations of the dwarf spheroidal galaxy Segue 1. Predictions are also made on improvement that can be achieved for MAGIC and CTA. Lastly, we discuss how this method can be applied in a global, sensitivity-optimized indirect Dark Matter search that combines the results of all Cherenkov observatories of the present generation.
\end{abstract}

The European Physical Society Conference on High Energy Physics -EPS-HEP2013

18-24 July 2013

Stockholm, Sweden

\footnotetext{
${ }^{*}$ Speaker.

$\dagger$ This work has been supported by Spanish MICINN through the programs FPA (grants FPA2009-07474 and FPA2010-22056-C06-01) and Consolider-Ingenio-2010 (grant MultiDark CSD2009-00064).
} 
Standard analysis in Imaging Air Cherenkov Telescopes (IACTs), such as MAGIC, VERITAS, H.E.S.S., or the forthcoming CTA, are usually optimized for sources with featureless spectral distributions, well described by a simple power law. We propose a dedicated approach, optimized for spectral features of Dark Matter (DM) origin.

In IACT standard analysis chain, the existence of a source is established by comparison of the number of events detected in the source region $(n)$ with the number of events detected from the control, background region(s) $(m)$. The number of gamma-ray $(g)$ and background $(b)$ events present in the source region can be hence estimated by maximization of the following likelihood function:

$$
\mathscr{L}(g, b \mid n, m)=\frac{(g+b)^{n}}{n !} e^{-(g+b)} \times \frac{(\tau b)^{m}}{m !} e^{-\tau b},
$$

referred here as the conventional likelihood approach, where $\tau$ is the normalization between the signal and background regions. The energy integration range can be optimized for different DM mass and annihilation/decay channels, as done, e.g. in [1].

We propose including the expected spectral shape in the maximum likelihood analysis. This full likelihood [2] function has, for a given DM model $M$ with parameters $\theta$, the following form:

$$
\mathscr{L}\left(N_{\mathrm{EST}}, M(\theta) \mid N_{\mathrm{OBS}}, E_{1}, \ldots, E_{N_{\mathrm{OBS}}}\right)=\frac{N_{\mathrm{EST}}{ }^{N_{\mathrm{OBS}}}}{N_{\mathrm{OBS}} !} e^{-N_{\mathrm{EST}}} \times \prod_{i=1}^{N_{\mathrm{OBS}}} \mathscr{P}\left(E_{i} ; M(\theta)\right),
$$

with $N_{\mathrm{OBS}}(=n+m)$ and $N_{\mathrm{EST}}$ denoting the total number of observed and estimated events, respectively. $\mathscr{P}\left(E_{i} ; M(\theta)\right)$ is the value of the probability density function (PDF) of the event $i$ with measured energy $E_{i}$, defined as:

$$
\mathscr{P}(E ; M(\theta))=1 / A \times P(E ; M(\theta)),
$$

where $A=\int_{E_{\min }}^{E_{\max }} P(E ; M(\theta)) d E$, and $E_{\min }$ and $E_{\max }$ are the lower and upper limits of the considered measured energy range. $P(E ; M(\theta))$ represents the differential rate of signal and background events, such that:

$$
P(E ; M(\theta))=\left\{\begin{array}{ll}
P_{B}\left(E_{i}\right), & i \in B \\
P_{S}\left(E_{i} ; M(\theta)\right), & i \in S
\end{array},\right.
$$

with $P_{B}(E)$ and $P_{S}(E ; M(\theta))$ being the expected differential rates from the background $(B)$ and source $(S)$ regions, respectively:

$$
P_{B}(E)=\tau \int_{0}^{\infty} \frac{d \Phi_{B}}{d E^{\prime}} R_{B}\left(E ; E^{\prime}\right) d E^{\prime}
$$

and

$$
P_{S}(E ; M(\theta))=\int_{0}^{\infty} \frac{d \Phi_{B}}{d E^{\prime}} R_{B}\left(E ; E^{\prime}\right) d E^{\prime}+\int_{0}^{\infty} \frac{d \Phi_{G}(M(\theta))}{d E^{\prime}} R_{G}\left(E ; E^{\prime}\right) d E^{\prime} .
$$

Here, $E^{\prime}$ denotes the true gamma-ray energy; $d \Phi_{B} / d E^{\prime}$ and $d \Phi_{G} / d E^{\prime}$ are the differential fluxes of cosmic (background) and gamma-ray (signal) radiations, and $R_{B}\left(E ; E^{\prime}\right)$ and $R_{G}\left(E ; E^{\prime}\right)$ are the telescope response functions to each of them, i.e. $R_{B, G}\left(E ; E^{\prime}\right)=A_{\mathrm{eff}_{B, G}}\left(E^{\prime}\right) G_{B, G}\left(E ; E^{\prime}\right)$, where $A_{\mathrm{eff}}\left(E^{\prime}\right)$ is the effective area and $G\left(E ; E^{\prime}\right)$ the energy reconstruction function. 

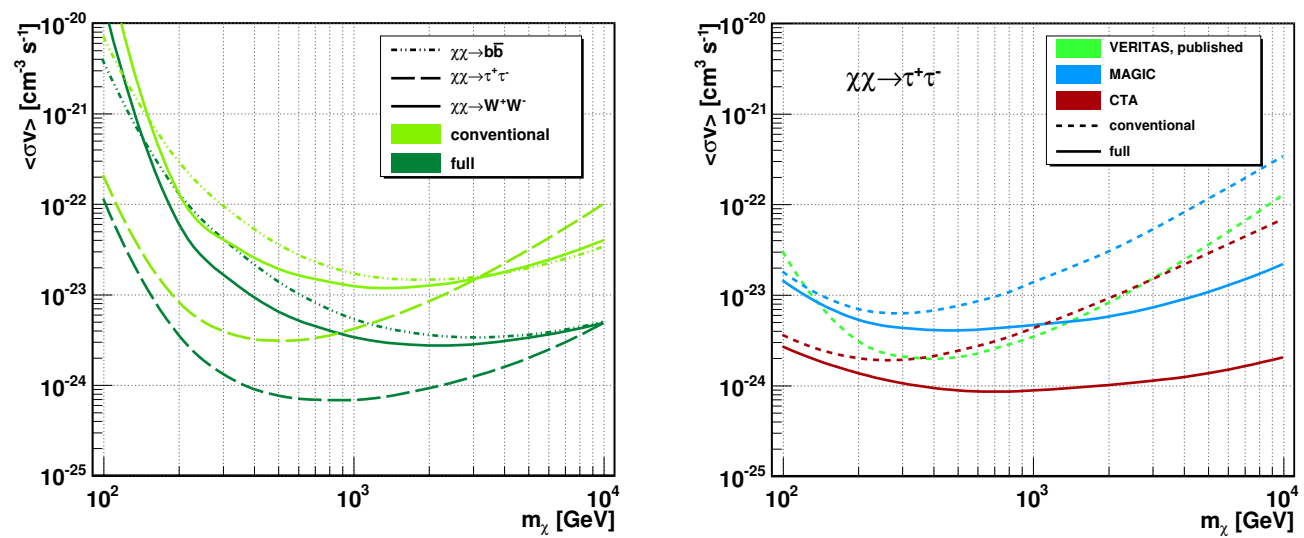

Figure 1: 95\% confidence level upper limits on $\langle\sigma v\rangle$ as a function of $m_{\chi}$, considering different annihilation channels, and $\sim 50 \mathrm{~h}$ of observations of the Segue 1 galaxy. Left: Results for VERITAS estimated by the conventional analysis (light green) and the full likelihood method (dark green). Right: Expected exclusion lines for $\chi \chi \rightarrow \tau^{+} \tau^{-}$, for MAGIC (blue) and CTA (red), obtained from both the conventional (dashed line) and full likelihood approaches (full line). Results from VERITAS, as given in [3], are also plotted for comparison purposes (green), although their validity is questioned in [2].

To estimate the performance of this new analysis approach we compare the limits on $\langle\sigma v\rangle$ recently published by VERITAS [3] with those that would had been obtained should the full likelihood analysis method had been used (see Figure 1-left). The more massive the DM particle, the greater the improvement, especially for the $\tau^{+} \tau^{-}$channel, whose spectrum gets harder for higher $m_{\chi}$ values, and the gain is larger than one order of magnitude. Additionally, we study the sensitivities of MAGIC [4] and CTA [5] for gamma-ray spectra from $b \bar{b}, \tau^{+} \tau^{-}$and $W^{+} W^{-}$annihilation channels. The obtained exclusion limits for the case of $\tau^{+} \tau^{-}$for both methods are shown on Figure 1-right. The constraints from the full likelihood method are more significantly improved with respect to those from the conventional analysis for the more massive DM particles, being higher (up to a factor $\sim 30$ ) for CTA, due to its better energy resolution.

The full likelihood method allows a rather straightforward combination of the results obtained by different instruments and from different targets. For a given DM model $M(\theta)$, and $N_{\text {inst }}$ different instruments (or measurements), a global likelihood function can be simply written as $\mathscr{L}_{T}(M(\theta))=$ $\prod_{i=1}^{N_{\text {inst }}} \mathscr{L}_{i}(M(\theta))$, which can be maximized in a rather trivial way. This approach eliminates the complexity required for a common treatment of data and response functions of different telescopes or analyses. Since DM signals are universal and do not depend on the observed target, the results from different sources can also be combined through the overall likelihood function, providing therefore a more sensitive DM search.

\section{References}

[1] J. Aleksić et al., JCAP 06 (2011) 035

[2] J. Aleksić, J. Rico and M. Martinez, JCAP 10 (2012) 032

[3] E. Aliu et al., Phys. Rev. D85 (2012) 062001

[4] J. Aleksić et al., Astropart. Phys. 35 (2012) 435

[5] M. Actis et al., Exp. Astron. 32 (2011) 193 